Lengua y Sociedad, revista de lingüística teórica y aplicada

Vol. 17, n. ${ }^{\circ}$ 2, Lima, julio-diciembre de 2018, pp. 9-25 https://doi.org/10.15381/lengsoc.v17i2.22350

\title{
Humor verbal en Pataclaun: el fenómeno de la paronomasia
}

\section{Verbal humour in Pataclaun: the phenomenon of paronomasy}

\author{
Mariana Mendoza Villanueva \\ Univ. Nacional Mayor de San Marcos \\ mariana.mendoza@unmsm.edu.pe
}

\author{
Lucero Paredes Castillo \\ Univ. Nacional Mayor de San Marcos \\ vania.paredes@unmsm.edu.pe
}

\begin{abstract}
Resumen
El artículo contiene una recopilación de algunos diálogos presentados en el programa Pataclaun (1997-1999) que permiten la visualización y explicación del fenómeno de la paronomasia, definido por López (2005) como un juego del lenguaje que involucra a fenómenos lingüísticos como la polisemia, paronimia, homonimia y resegmentación. La paronomasia se configura a través de diversos fenómenos lingüísticos, como la polisemia y homonimia. A partir de lo anterior, en el artículo se concluye que la paronomasia es un concepto aplicable para un estudio de las características del humor verbal del programa Pataclaun.
\end{abstract}

Palabras claves: Humor verbal, juego lingüístico, paronomasia, polisemia, homonimia. paronimia

\begin{abstract}
The article contains a compilation of some dialogues presented in the Pataclaun program (19971999) that allow the visualization and explanation of the phenomenon of paronomasia, defined by López (2005) as a language game that involves linguistic phenomena such as polysemy, homonymy and resegmentation. Paronomasia is configured through various linguistic phenomena, such as polysemy and homonymy. From the above, the article concludes that paronomasia is an applicable concept for a study of the characteristics of verbal humor in the Pataclaun program.
\end{abstract}

Keywords: Verbal humour, linguistic game, paronomasia, polysemy, homonymy. 


\section{Introducción}

A lo largo del tiempo, los juegos de palabras han sido parte de nuestra vida cotidiana. Cada juego originado con la elección de una palabra que posee una variedad de significados, pero una contrastiva simplicidad respecto a su sonido. Este tipo de palabras, denominadas parónimas, indicaban el manejo de la lengua y un mayor conocimiento del vínculo de la lengua con la realidad.

Con el paso del tiempo, este juego de palabras ha pasado a ser considerado como un juego lingüístico, pero dicha propuesta carece de fundamentos a pesar de la existencia de una tesis que versa sobre los juegos lingüísticos en el español, lo que hace que hasta la actualidad no exista una denominación específica de juegos lingüísticos. Sin embargo, en el estudio de María Moliner, en la especificación de juegos lingüísticos, se introduce el término paronomasia que ella define como: «chiste conseguido manejando palabras parónimas». Del mismo hace referencia a los juegos del lenguaje; los cuales están presentes en toda cultura, es decir, son universales e inagotables ya que transmiten de una forma humorística los sucesos de la realidad y su conexión con el mundo de las ideas (C. López, 2005).

Para dichos juegos uno de los elementos más esenciales, como ya se ha mencionado anteriormente, es el uso de la paranomasia, ya que está presente en diversos procesos lingüísticos estandarizados o no estandarizados, como en la formación de un chiste, una adivinanza o un trabalenguas. Dentro de los estudios de Apreijan y Mel Ck (2001), se traza un nuevo enfoque de la definición de paronomasia dentro del marco teórico del lexicón mental, el cual indica que a pesar de que el lexicón poseía una estructura cohesiva podía permitir una descripción ad-hoc.

En la actualidad, con el avance y desarrollo de la tecnología, se puede observar que los juegos del lenguaje son un recurso dentro de la participación humorística presentado tanto en los programas televisivos o radiales como en series televisivas y revistas en los cuales dentro de su contenido posee un distintivo modo de presentar o comentar diversos temas de la realidad particularmente políticos y de espectáculo resaltando de este su lado cómico, risueño o ridículo. Este tipo de presentación además añade el uso de ciertas figuras o también llamadas técnicas específicas. En el presente artículo nos enfocamos en describir una de estas técnicas, la paronomasia; se realiza una distinción entre las formas de paronomasia potencial y paranomasia humorística. También se hace mayor énfasis en la última a pesar de que entre ambas las formas afines por su significante o su significado son establecidas y reconocidas por el hablante al momento de ejecutarse el juego verbal.

10 Lengua y Sociedad 
Además, se describirá como la paronomasia es partícipe en la creación del humor verbal propio del programa televisivo Pataclaun, a través de los juegos de lenguaje, producido por los personajes principales: Wendy y Machín (los cónyuges), Monchi (la bebé) y el trío Queca, Gonzalete y Tony (los fantasmas); como también por los personajes secundarios o extras tales como: Antonieta (la mamá de Tony), Don Carlos (papá de Machín), Janeth Wendy (hermana de Wendy) Jhonatan (clon de Gonzalete), Sheyla (pariente lejano de Queca) entre otros participantes de la serie televisiva peruana que desarrolla las aventuras de tres fantasmas junto a la pareja conformada por Machín y Wendy. Además, la serie poseía características particulares como el uso de modismos y expresiones propias de la jerga peruana. Mantuvo una buena acogida por parte del público hasta su cancelación y posteriormente, los actores se involucraron en proyectos relacionados al mundo claun. La serie fue transmitida de 1997 hasta 1999 a través de Frecuencia Latina y en la actualidad es transmitida por Movistar Series.

Con la finalidad de comprender el funcionamiento de la paronomasia en la configuración del humor verbal de dicho programa, así como también describir y clasificar sus tipos. Puesto que la paronomasia implica dentro del hablante una capacidad para el entendimiento y creación de los ya mencionados juegos de palabras basados en fenómenos como la polisemia u homonimia, los cuales llegarían a ser los tipos de paranomasia presentes en el español, lo que hace que se pueda considerar a dicha capacidad un tipo de competencia a la que se le denominaría competencia paronomásica la cual es acumulativa (tal como la competencia lingüística) y permite tener un mayor conocimiento de la lengua y sus posibilidades paronomásicas.

Para ello, se realizará un análisis de un corpus que se constituye a partir de la recopilación de los diálogos emitidos en la serie televisiva. Primero, se procede a identificar el tipo de paronomasia en cada uno. Luego, se explica la configuración de la paronomasia. La estructura del trabajo consiste primero en la exposición del planteamiento del problema de investigación con la respectiva hipótesis, en segundo lugar, se presentarán los objetivos generales y específicos. Luego, se colocará los diálogos escogidos (corpus) para el análisis y por último se expondrán las conclusiones. 


\section{Humor verbal y chiste lingüístico}

Sobre el humor verbal, los aportes de Attardo constituyen uno de los pocos trabajos desde una perspectiva lingüística. Uno de los aspectos más importantes, planteados por este autor, es la inclusión de la pragmática en la interpretación del fenómeno humorístico. Así, Attardo (citado en Torres, 1997) plantea una distinción entre lo que ocasiona risa, que denomina émico, y lo que los teóricos consideran divertido en función de la intención creadora y las características estructurales que poseen las manifestaciones lingüísticas del humor. Además, insiste en la competencia, como conocimiento tácito, como la clave del humor (p. 436).

Otro punto tratado por la Teoría General del Humor Verbal de Attardo es el tratamiento de los chistes y los puns. Por un lado, los puns son considerados como casos de homónimos, metáforas o expresiones proverbiales. En el caso de los chistes, estos se incluyen en el conjunto general de textos narrativos. En este sentido, los caracteriza como texto desviado, es decir, como texto que viola el principio de cooperación que se presenta en una conversación seria. Sin embargo, como se puede observar en la realidad, si se dan los intercambios humorísticos a pesar de la violación del principio antes mencionado. En consecuencia, Raskin (citado en Torres, 1997) plantea la existencia de otro principio de cooperación que guía la comunicación no sería que es aplicable a los chistes u otros textos afines (pp. 444-445). Finalmente, para Attardo y Raskin, una teoría que pretenda estudiar

el humor debe considerar a este como un fenómeno que integra la estructura lingüística dentro de los contextos sociales y culturales del uso del lenguaje. En este sentido, el humor verbal se concibe como algo culturalmente específico (Jiménez, 2016, p. 30).

Según lo anteriormente mencionado, el chiste es un tipo de manifestación lingüística de humor. En este sentido, para Luque (2007, p. 92) el chiste lingüístico es una manipulación de materiales lingüísticos para conseguir un efecto de sorpresa o risa en el hablante. En este sentido, señala que el chiste tiene como base a errores lingüísticos y fallas en la comunicación. Además, enfatiza la importancia cultural en la configuración y el funcionamiento de este tipo de expresiones.

\section{La paronomasia}

Es un juego del lenguaje que vincula a diferentes fenómenos lingüísticos. Presenta tres tipos: 1) paronomasia por los múltiples significados (polisemia); 2) 
paronomasia por coincidencia total o parcial de las formas (homonimia), 3) paronomasia por posibilidad de resegmentar el discurso (resegmentación).

De esta manera, se nota que, la polisemia, la homonimia y la ambigüedad estructural son los fenómenos más recurrentes en la configuración de la paronomasia. Además, se menciona la existencia de diferentes términos designar los juegos lingüísticos. En este sentido, opta por el uso del término paronomasia para incluir a diversos tipos de juegos del lenguaje (López, 2005).

También, se presenta la consideración de la paronomasia desde el marco teórico del lexicón mental, que surge como una guía para la investigación del léxico y su relación con la forma en que los hablantes relacionan conceptos entrelazados con diferentes relaciones semánticas. Para un mejor entendimiento de esto, se introduce el concepto de mapa mental. Este concepto permite dilucidar aspectos de las conexiones y relaciones indirectas entre las palabras debido a que denotan realidades organizadas en la mente del hablante (López, 2005, pp. 80-86).

A continuación, se presentan los conceptos de polisemia y homonimia, ya que son los fenómenos lingüísticos encontrados en el humor verbal del programa Pataclaun. Para Escandell (2007, p. 37) estos son tipos de ambigüedad léxica, es decir, son palabras poseen más de un significado en diferentes contextos.

\subsection{Polisemia y homonimia}

Según la RAE, la polisemia se define como "pluralidad de significados de una expresión lingüística». Además, Ullmann (1965) indica que la polisemia era un recurso para desorientar a los oyentes. También, apunta que surge a partir de la metáfora y la metonimia. Asimismo, para Escandell (2007) es el fenómeno que se produce cuando una palabra expresa significados diferentes, aunque relacionados (p.41). En el mismo sentido, Cuenca y Hilferty (2013) mencionan, desde la perspectiva de la lingüística cognitiva, que el requisito mínimo para hablar de polisemia es la referencia a dos sentidos diferentes pero interrelacionados (p. 125). Esto es un rasgo que la diferencia del fenómeno de la homonimia.

Los tipos de polisemia considerados por López (2005, p.89) son los siguientes: 1) polisemia léxica: palabra que poseen dos o más sentidos. Se trata de la polisemia propiamente dicha; 2) polisemia sintáctica: son construcciones cuya interpretación varía según el punto de vista del espectador u oyente. Esta última se estudia dentro de la ambigüedad estructural. 
En el caso de la homonimia, definida por la RAE como la relación entre homónimos que presenta las siguientes formas: 1) homofonía: coincidencia en la pronunciación de dos palabras, escritos de diferente forma y poseen distinto significado; 2) homografía: coincidencia en la pronunciación de dos palabras que se escriben de forma diferente y tienen distinto significado. También, menciona Escandell (2007) que, es el fenómeno que se produce cuando hay igualdad en la forma de dos o más palabras con significados diferentes y no relacionados. Asimismo, plantea que no hay un límite claro entre polisemia y homonimia (p. 41).

\section{Metodología}

El análisis de la frecuencia del uso de la paranomasia y sus tipos que se realiza en el presente artículo es de tipo cualitativo. El análisis se realiza en base a los diálogos que son producto de la interacción entre los personajes de la serie televisiva peruana Pataclaun. Cada capítulo tiene una duración que varía entre 40 a 45 minutos. A partir de esto, se realizó una recopilación de diálogos efectuados a lo largo de sus dos temporadas, ya sea de forma improvisada o ensayada, dentro de un determinado contexto, tanto en forma explícita o implícita.

A partir de lo anterior, se realizó una breve descripción y se reconoció el uso de la paranomasia para la creación del texto o intervención verbal humorística. Además, de acuerdo con cada diálogo, se identificó el tipo de paronomasia con su respectiva descripción y evaluación del caso, colocando las palabras resaltadas que presentan acepciones que hacen posible el chiste lingüístico.

\section{Análisis de la paronomasia en el humor verbal de Pataclaun}

\subsection{Casos de polisemia}

\subsubsection{Acepciones particulares}

Para que se haga visible el fenómeno de la paronomasia de este tipo, en los siguientes diálogos se presentan algunas acepciones que solo pueden ser reconocidas en Perú (país de origen del programa).

\section{Lengua y Sociedad}


Humor verbal en Pataclaun: el fenómeno de la paronomasia

Tony: ¿quién descubrió América?

Machín: Rulito Pinasco

(Capítulo: Hombres vs Mujeres)

En este diálogo, el chiste lingüístico es posible si los televidentes residen en Perú y tienen un conocimiento mínimo del ámbito de los medios de comunicación del país. Esto se explica de la siguiente manera: la palabra América hace referencia a América Televisión, nombre del canal peruano de televisión abierta (canal o4) y no al continente perteneciente al hemisferio occidental del planeta.

Monchi: Tío Tony ¿tú eres cholo?

Tony: mamita por favor no me interrumpas cuando estoy grabando, por favor Monchi: solo contéstame

Tony: es que yo te tengo que explicar que estoy mal de los nervios, estoy con pastillas, estoy un poco loco.

Monchi: solo contéstame si eres cholo y me voy

Tony: mamita, no soy cholo. Soy villano, soy malo.

(Capítulo: Miss Perú)

Dentro de este diálogo para el análisis del uso de la palabra cholo, se requiere la participación de un enfoque literario puesto que dentro de la literatura peruana se hace uso del término cholo para referirse al personaje benévolo dentro de una historia o relato. De acuerdo a ello en este dialogo, la palabra cholo, no hace referencia a cuestiones raciales discriminatorias arraigadas en la misógina anticultura que comparte sociedad latinoamericana sino al héroe o personaje central sobre el cual pivota la trama de las obras de la narrativa peruanista, puesto que en dicho dialogo el personaje Tony recalca no ser cholo (es decir el héroe de la historia) sino el villano lo opuesto del arquetipo de héroe que representa la palabra cholo.

Tony: el curita sólo duerme con sus hostias y su cáliz

Machín: Ah sí y su rosario...

Tony: su rosario... la Rosario Pérez, la patrona, la última.

(Capítulo: Queca y Gonzalete se casan)

Para este diálogo la paranomasia se hace presente a través de la polisemia ya que la palabra rosario no expresa su significado de 'emblema religioso conformado por una serie de cuentas ensartadas y separadas de diez en diez por otras de distinto tamaño, usado para la oración que realizan los católicos para recordar 
los misterios de la vida, de la muerte y resurrección de Jesús'. Se refiere, más bien, a un personaje de espectáculos llamada Rosario Pérez popular por causar cierta revolución entre el género masculino en el periodo de difusión de este programa. La transcripción hace más evidente la diferencia de ambos términos puesto que, en el lenguaje hablado, hacer mención a una palabra que no lleve o difiera por una fuerza mayor de voz dificulta la interpretación de saber si se trata de una palabra común o un nombre propio_como en este caso_y ayuda a la realización del chiste.

Machín: A mí que me importa [..]. Este es mi teléfono y nadie va a hablar por teléfono. Sea acabo.

Queca: A ver, discúlpame, ¿ya terminaste?

Machín: No. Me faltó segundo de primaria [todos ríen]

(Capítulo: Los inquilinos)

En este caso, el personaje Queca hace uso de la palabra terminaste para preguntar al personaje Machín si ya acabó de realizar su llamada con el otro operador. Por su parte, el personaje Machín le otorga a dicha interrogación el significado de haber completado todos los niveles de sus estudios, es decir, nivel inicial, primario, secundario, preparatoria, superiores (universitarios) y otros.

\subsubsection{Connotación sexual}

Un aspecto resaltante del humor verbal del programa son los chistes de contenido sexual. En estos casos, para que el chiste funcione, el oyente debe conocer los significados a los que los hablantes, en este caso los actores, hacen referencia como se puede observar en los siguientes casos:

Queca: ¡Tony! ¡Tony!

Don Carlos: para que quieres a Tony si acá tengo a tu muñequito.

(Capítulo: Los cuernos)

En este diálogo, el personaje Don Carlos hace uso de la palabra muñequito para referirse de manera informal, mediante una expresión jergal, a su miembro viril $\mathrm{u}$ órgano reproductor masculino, es decir, su pene. No significa lo que señala el diccionario de la RAE como: figura de persona hecha generalmente de plástico, trapo o goma, que sirve de juguete o de adorno. Ni tampoco se refiere, dentro del ámbito medicinal, a aquella pieza pequeña de trapo que, atada con un hilo por

16 Lengua y Sociedad 
las puntas, encierra algún ingrediente o una sustancia medicinal que no se debe mezclar con el líquido que se cuece o empapa.

Otro ejemplo del mismo tipo es el siguiente:

Wendy: Tony, Tony estás mojado.

Tony: Si eso pasa cuando te veo.

(Capítulo: La rata)

En este diálogo, el uso de la palabra mojado hace referencia al acto de lubricación previo a consumar el coito realizado por el órgano reproductor masculino al encontrarse en estado de excitación, el cual dentro del diálogo se sobreentiende que es desencadenado por el personaje Wendy.

Machín: No vas a donar mi pelota. Esa es mi pelota. ¡Mi pelota!

Wendy: Pero Machín tú tienes muchas pelotas...Eres bien pelotudo.

(Capítulo: Machín no se baña)

En este caso la palabra pelotudo no hace referencia a una persona que tiene en su posesión un conjunto de pelotas, es decir, una cantidad abundante de un objeto utilizado en los deportes o actividades lúdicas. Además, en el diccionario de peruanismos, se califica dicho término como el adjetivo huevón.

Para este diálogo, dicha expresión jergal busca referirse de manera informal a las dos glándulas sexuales masculinas, de forma oval, que segregan los espermatozoides. Ello, debido a la semejanza física que existe entre la palabra pelota, de forma esférica u ovaladas y una dura consistencia, con la palabra de testículo que posee una forma de ovoide aplanado en sentido transversal con una consistencia dura y algo elástica.

Machin: Somos malos, curita, somos malos.

Gonzalete: yo tengo la solución [...] Hay que cambiar de giro nuestras vidas.

Hay que ser hombres nuevos, pasarnos al otro equipo.

Machin: (sonríe) Asuuu, curita yo ya estoy viejo para esas cosas.

Gonzalete: Oyeeee, no, no, noooo [...] Yo me refiero, hay que pasarnos al equipo de los buenos.

(Capítulo: La bolsa de la caridad)

Dentro de este diálogo la paranomasia se hace presente en el uso de la palabra equipo donde no expresa literalmente equipos, es decir bandos o grupos de 
personas que son participes de alguna actividad didáctica o física dentro de una competencia sino a la contraparte de la especificación de la orientación sexual ya sea homosexuales o bisexuales.

$[\ldots]$

Tony: Wendy Janet, a la Micky mouse de micro que la quiero, que la quiero mucho, que la deseo.

Tony: Que quiero tener como soy fantasma muchas penitas con ella. Un ratito...

(Capítulo: Wendy está embarazada)

Para este diálogo el uso de la palabra penitas no posee el significado del conjunto de pesadumbres o tristezas en su forma diminutiva, en este contexto el personaje Tony le da el significado de connotación sexual para referirse a las ganas acumuladas que tiene de satisfacer su deseo sexual con el personaje Wendy.

[...]

Queca: A encima una acá sola, una niña dulce, virgen, pura y casta

Don Alberto: Jajajajajajaa

Queca: Que se ríen

Don Alberto: Una niña virgen, pura y casta [riéndose]

Queca: Así es, con un hombre depravado y ansioso de sesos

Don Alberto: Bueno eso sí

Queca: Dios mío que va a decir la gente de mí

Don Alberto: la gente no tiene que decir nada

Queca: ¿Dónde está la bruja? Perdón su esposa

Don Alberto: ¿Quién?

Queca: Chabela

Don Alberto: ahh ¿Chabelita? Chabelita, este... se fue a la procesión. Así que tiene por lo menos un par de horas.

Queca: Ohhh

Don Alberto: Pero aquí me tienes a mi mamita linda, bien bañadito y con los aretes bien talqueados.

(Capítulo: Centro de Lima)

Aquí, el uso de la palabra aretes no posee el significado de aquel objeto que es parte del conjunto de las joyerías, que sirve para adornar parte del cuerpo y en este caso es ubicado en las orejas también se le conoce como pendientes. Por lo

18 Lengua y Sociedad 
contrario, el personaje de Don Alberto usa esta expresión para hacer referencia a sus testículos, parte de su órgano reproductor.

\subsubsection{Apodos}

Otra particularidad del humor verbal del programa es la presentación de chistes a partir de los apodos. Según el DRAE, un apodo es «Nombre que suele darse a una persona, tomado de sus defectos corporales o de alguna otra circunstancia». En este sentido, en el programa, los apodos se basan en la apariencia, la procedencia, la educación, la vida profesional de cada uno de los integrantes. Hay que precisar que algunos apodos refieren información muy específica sobre el integrante en mención y por esto no han sido referidos.

Todos: ¡Wendy!, ¡Wendy! [...]. ¡Hay una muca en la casa!

[Wendy viene corriendo].

Wendy: Claro pues, si no me sacas a pasear, ¿dónde quieres que esté? [ríen].

Tony: Un ratito, un ratito. Nosotros estamos hablando de una muca de verdad [...].

(Capítulo: Centro de Lima)

El caso anterior presenta un chiste basado en el apodo recurrente de Muca que se le da al personaje Wendy. Entonces, la palabra muca que el resto de los personajes utiliza para referirse a una rata de gran tamaño, para el personaje Wendy hace referencia a ella misma.

Queca: [...] En este caso, el Ken eres tú (lo mira), bueno, salvaguardando las distancias.

Tony: ¿Qué te pasa? ¿Qué te pasa? Soy un muñeco. Muñeca, soy un muñeco. Queca: Bueno, un muñeco bien feo.

$$
\text { (Capítulo: El deseo de Gonzalete) }
$$

En el programa es recurrente que el personaje Queca llame al personaje Tony con este apodo, tal vez en forma irónica. En este caso, la palabra muñeca y muñeco son utilizadas para referirse a una persona con buena apariencia física. Además, la palabra muñeco también tiene el significado, según el DRAE de 1. figura de persona hecha de plástico o trapo. Es este último el significado utilizado por el 
personaje Queca, al que le agrega la frase ‘bien feo' para referir lo poco atractivo que resulta Tony para Queca.

[Después de ser detenido por Wendy].

Machín: ya pues, ¿Cómo me vas a poner una papeleta a mí? Wendy soy yo, mírame soy yo, soy Machín, tu esposo.

Wendy: Yo estoy casada con la ley.

Machín: ¡Maldita! Me sacaste la vuelta.

Wendy: Siiiiiii [ríen].

(Capítulo: Wendy policía de tránsito)

En este diálogo el personaje Wendy hace uso de la palabra casada para referirse al hecho de estar comprometida mediante un carácter ético en lo político o lo social, en este caso a respetar y a hacer cumplir con lo que dicta las leyes de tránsito. En cambio, el personaje Machín le da el significado del estado civil que adquiere una persona en el momento que contrae matrimonio, por ello dicho personaje hace la suposición que Wendy le ha sido infiel.

Tony: Chochera todo esto no te parece muy raro.

Machín: Oye aquí el único raro eres tú muñeco tumba latas.

Tony: Que te pasa guaipe de chongo (risas).

Tony: Que te pasa, viruta tu mujercita no te parece muy raro que en estos momentos se haga la sonámbula.

Machín: Se haga ¿Qué?

Tony: Sonámbula viruta, tu mujercita alguna vez ha sido sonámbula ¿ahh?

Machín: No ella es católica (risas).

(Capítulo: Wendy se lo comió todo)

En este diálogo, el personaje Tony hace uso de la palabra sonámbula para referirse la persona que desarrolla actividades motoras automáticas mientras permanecen inconscientes y sin la probabilidad de hablar, por ejemplo, un individuo puede salir de la cama, caminar, hacer sus necesidades e incluso salir de su casa. Mientras que el personaje Machín le da el significado de miembro de alguna religión, razón por la cual aclara que el personaje Wendy profesa el catolicismo, es decir, pertenece a la iglesia católica. 
$[\ldots]$

Queca: ¡Yo lavar!, ¡Queca lavar! El detergente percude mis manitas.

Don Alberto: Pero Queca [...] Como una mujer de alcurnia como tú, con ese cuerpito de sirena, de sirena... de camión.

Queca: [ríe].

(Capítulo: Wendy se rebela)

El personaje Don Alberto trata de halagar y burlarse del personaje Queca, de esta forma, la palabra sirena - cuya acepción coloquial es una mujer de buen aspecto físico-es emitida en primer lugar como un halago. Sin embargo, la palabra da un giro cuando se le agrega la frase de camión (hay que considerar que Queca es frecuentemente molestada por su apariencia física robusta) que provoca el cambio del significado de la primera palabra para así obtener el significado de objeto esférico y de gran proporción (en algunos casos).

\subsubsection{Jergas}

Dentro del humor verbal del programa también se hace la presentación de chistes utilizando una variedad lingüística del habla diferente de la lengua estándar. Esta estrategia es usada con frecuencia por distintos grupos sociales con la finalidad de ocultar el verdadero significado para su conveniencia o necesidad.

Wendy: Tony...

Tony: Ohh si, cosita rica.

Wendy: Tony ya estoy lista te espero.

Tony: Uhh No hay que hacer esperar a una dama.

Tony: Allá van mis tenazas, moñuda.

Tony: Uhh cosita rica, ya llego tu turroncito de maní.

Machín (disfrazado de Wendy): Yo te voy a dar tu turroncito de maguey.

(Capítulo: Los Cuernos)

En este diálogo, se hace uso de la expresión jergal tenazas, que no hace referencia al utensilio o herramienta que sirve para agarrar o sujetar cosas, sino que guarda una relación directa con las funciones que realizan las manos. Por ello, dentro de este diálogo se reemplaza la palabra manos por dicha expresión jergal. 
$[\ldots]$
Tony: ¿cuántas patas tiene un perro?
Machín: No sé, mi perro no tiene amigos

(Capítulo: Hombres vs. Mujeres)

Aquí, el personaje Machín vuelve a darle una acepción adicional a la palabra patas- que para el personaje Tony significa pie o pierna de un animal - que también tiene el significado de amigos. Este significado es mencionado en el Diccionario de Peruanismos como «pata: 1. Coloq. Amigo»

Doña Antonieta: ¡Que linda mi nuera! [...]

Doña Antonieta: Me gane con mi nuera. (Mira y acaricia a Queca).

Doña Antonieta: Tú también has estado tomando levadura. (Señala a Tony).

Desde chiquito le ha gustado el panetón.

(Capítulo: Machín no se baña)

En este caso, la palabra panetón, que significa - según el diccionario- «bollo hecho con una masa de tipo brioche, pasas y frutas confitadas o cristalizadas», es utilizada por el personaje doña Antonieta para referirse a la contextura física robusta de Queca. Esto también se entiende, a partir de su anterior línea cuando dice «has estado tomando levadura» (es un ingrediente del panetón).

Doña Antonieta: No, no, i¿qué?! [...]

Doña Antonieta: No me vengan a dar sinsabores en este momento, que yo ya estoy con los crespos hechos, si no mira... (se voltea y señala su cabello) los crespos hechos para la boda.

(Capítulo: Machín no se baña)

En este diálogo el uso del calificativo crespos no hace referencia al adjetivo para los cabellos rizados que se ha hecho el personaje de Doña Antonieta, sino en dicho contexto se le da el significado de los hechos ya concluidos para determinada celebración.

[Wendy se retira]

Tony: Wendy del hortelano. No come, ni deja que se la coman.

(Capítulo: Lorenita Rivasplata)

Aquí, el personaje Tony cambia las palabras del refrán «como el perro del hortelano, no come, ni deja comer» para referirse al personaje Wendy que primero 
coquetea con él y le pide que no vaya a su cita romántica con una mujer y luego lo deja solo. Entonces, se entiende que con la palabra comer el personaje utiliza un eufemismo para el acto del coito ya que es una expresión que sustituye otra más vulgar (tales como: cojan, tiren, follen, agarren, copulen, entre otros).

Tony: Queca se está haciendo, se está haciendo.

Don Carlos: Oh entonces habrá que limpiarla

(Capítulo: Los cuernos)

En este diálogo el personaje Tony utiliza la palabra haciendo para referirse a que el personaje Queca está fingiendo, es decir presenta una actitud distinta de modo que parezca real. Por su parte, el personaje Don Carlos percibe la palabra haciendo como el proceso biológico de expulsar, eliminar o expeler los excrementos o heces por el ano.

\subsection{Casos de paronimia y homonimia}

Queca: Dios mío Wendy, estoy completamente desvastada, desvastada.

Wendy: Devastada (sonríe), o, ¿ se te ha salido la basta?

Queca: [ríe] Nooo. Devastada, completamente humillada.

(Capítulo: Queca y Gonzalete se casan)

En este ejemplo (que se trata de una equivocación en la pronunciación del personaje Queca), se relacionan las palabras desbastada y devastada que tienen parecida pronunciación. Ahora, el personaje Wendy le da un giro a la situación cuando corrige la palabra mal pronunciada por el personaje Queca (desvastada), pronunciándola correctamente (devastada) y le pregunta si lo que quería decir era la palabra desbastada, que en el castellano de Lima puede entenderse con el significado de 'estar con la basta descosida' (esto ocurre en el momento que dice «o ¿se te ha salido la basta?») y de esta forma se configura el chiste lingüístico a partir de las palabras parónimas devastada y desbastada.

Hay que considerar que, desde la perspectiva de diferentes autores, la polisemia y homonimia se presenta como fenómenos diferentes, sin embargo, también se señala que no hay una línea nítida de distinción entre ambos. Debido a esto citamos un diálogo referido anteriormente para representar el fenómeno de la homonimia: 
Tony: el curita sólo duerme con sus hostias y su cáliz

Machin: Ah sí y su rosario...

Tony: su rosario... la Rosario Pérez, la patrona, la última.

(Capítulo: Queca y Gonzalete se casan)

Como se puede ver en el diálogo anterior, la palabra rosario- que significa: 1. Objeto formado por una serie de cuentas ensartadas y separadas [...] que se usa para rezar- es trasladada al nombre femenino Rosario. Entonces, se produce un cambio de significado entre las palabras homónimas rosario y Rosario.

\section{Conclusiones}

La paronomasia, concepto que engloba a diferentes juegos lingüísticos y que involucra a fenómenos como la polisemia, homonimia, paronimia y resegmentación, ayuda a comprender la conformación del humor verbal del programa Pataclaun. Como ya se mencionó, el programa se caracterizó por presentar guiones no tan estrictos, ya que la espontaneidad era parte fundamental dentro del elenco artístico de la serie Pataclaun.

La frecuencia del uso de la paranomasia en el humor verbal de la serie resulta mayor en su forma polisémica ya que dentro del análisis se pueden identificar más diálogos que recurren a la polisemia para la configuración del chiste con el toque humorístico debidamente contextualizado. Dentro de la serie no se logrado identificar una cantidad similar de ejemplos del uso de la homonimia ya que esta no resulta favorable para el elenco.

Un aspecto relevante en la configuración del humor verbal y, en específico, del chiste lingüístico es la competencia pragmática del hablante/oyente en la interacción que se da mediante el diálogo. Así, los personajes emiten sus diálogos con una clara intención, que es provocar risa. Para lograr esto, el hablante (el interlocutor, es decir el otro personaje) y el oyente (el público televidente) deben poseer conocimientos compartidos que hagan posible el entendimiento del chiste lingüístico.

Pataclaun surge como una propuesta televisiva diferente ya que utiliza el humor de una forma audaz que, creemos, se debe en mayor medida al uso de la paronomasia. Esto último se convierte en un rasgo distintivo de los diálogos presentados en el programa y que demuestran el ingenio de su equipo de escritores.

24 Lengua y Sociedad 
Humor verbal en Pataclaun: el fenómeno de la paronomasia

\section{Bibliografía}

Alvarez, J. (2009). Diccionario de peruanismos. El habla castellana del Perú. Recuperado de http://repositorio.uap.edu.pe/bitstream/uap/4112/1/diccionario_de_peruanismos.pdf

Andreína, M. (2012). Polisemia regular y representación lexicográfica: los nombres locativos en español. Articles, 5, 34-38. Recuperado de https://www.raco.cat/ index.php/Terminalia/article/viewFile/257904/345152

Attardo, S. (2001). Humorous Texts: A Semantic and Pragmatic Analysis. Berlín: Mouton de Gruyte.

Cuenca, M., \& Hilferty, J. (2013). Introducción a la lingüística cognitiva. España: Ariel.

Escandell, M. (2007). Apuntes de Semántica léxica. España: UNED.

Jiménez, N. (2016). La Teoría General del Humor Verbal en The breif wondrous life of Oscar Wao y La breve y maravillosa vida de Oscar Wao. Verbum et Lingua, 8.

Llera, J. (2004). La investigación en torno al humor verbal. La teoría frente a la hidra humorística. Revista de literatura, 66(132), 527-535. Recuperado de: http://revistadeliteratura.revistas.csic.es/index.php/revistadeliteratura/article/ download/132/143

López, M. (2005). La paronomasia como recurso conceptual, expresivo y humorístico en la lengua española actual (Tesis doctoral). Universidad de Granada, Granada. Recuperado de https://hera.ugr.es/tesisugr/15512149.pdf

Luque Durán J. D. (2007). Los juegos lingüísticos: fallos comunicacionales, humorismo verbal y reflexión metalingüística. Léxico español actual: Actas del I Congreso internacional de léxico español actual, 91-126.

Naters, J. (Productor). (1997-1999). Pataclaun [Serie de televisión]. Lima- Perú: Asociación cultural Pataclaun, Frecuencia Latina.

Pérez, J. y Merino, M. (2009). Definición de jerga. Recuperado de https://definicion.de/jerga/

RAE (2019). Diccionario de la Real Academia Española.

Ruiz, L. (2012). La lingüística del humor en español. Madrid: Arco/Libros.

Torres, M. (1997). Teorías lingüísticas del humor verbal. Pragmalingüistíca, 5-6, 435-448. doi: https://doi.org/10.25267/Pragmalinguistica.2017.i25

Ullman, S. (1976). Semántica. Introducción a la ciencia del significado. Madrid: Aguilar.

Valbuena de la Fuente, F. (2002). Humor verbal y humor de situación. Cuadernos de Información y Comunicación, 7, 381-383. Recuperado de: https://revistas. ucm.es/index.php/ciyc/article/view/ciyco202110381a 